\title{
The Attitudes, Feelings, and Experiences of Online Gamers: A Qualitative Analysis
}

\author{
Zaheer Hussain, M.Sc. and Mark D. Griffiths, Ph.D.
}

\begin{abstract}
The playing of massively multiplayer online role-playing games (MMORPGs) is now a highly popular leisure activity. The present study set out to explore the attitudes, experiences, and feelings of online gamers. The study entailed 71 interviews with online gamers (52 males, 19 females) from 11 different countries. Six main themes emerged from the analyses of the interview transcripts: (a) online gaming and integration into day-to-day lives; (b) online gaming, excessive play, and problems; (c) addiction; (d) psychosocial impact of online gaming; (e) online gaming, dissociation, and time loss; and (f) online gaming and the alleviation of negative feelings and mood states. These findings specifically showed how gamers used MMORPGs to alleviate negative feelings and provided detailed descriptions of personal problems that had arisen due to playing MMORPGs. The implications of these findings are discussed in relation to previous qualitative and quantitative research in the area.
\end{abstract}

\section{Introduction}

$\mathbf{R}$ ESEARCH IN THE AREA of massively multiplayer online role-playing games (MMORPGs) has been growing rapidly. Research has focused on the demographic makeup of gamers, ${ }^{1,2,3}$ group membership and guild dynamics, ${ }^{4}$ social motivations for playing and development of in-game friendships, ${ }^{5}$ motivations of gamers, ${ }^{6}$ online gaming addiction, ${ }^{7}$ and gender swapping. ${ }^{8}$ Griffiths et al. ${ }^{2}$ gathered primary data on the basic demographic factors of online game players. The results showed that $81 \%$ of online gamers were male and that the mean age of players was 28 years. It was also found that $4 \%$ of players claimed to play for over 70 hours a week. Yee's ${ }^{3}$ demographic survey of online gamers revealed similar findings.

Hussain and Griffiths ${ }^{8}$ investigated online socialization and the phenomenon of gender swapping in MMORPGs. Results showed that just over 1 in 5 gamers (21\%) preferred socializing online to offline. Gamers saw the online worlds as pleasant and satisfying environments that provide equality. Results also showed that $57 \%$ of gamers had engaged in gender swapping. Gamers' gender swapped for reasons of interest, to experiment playing a different gendered character, for fun, and to prevent unsolicited male approaches. Experimental research by Lim and Lee ${ }^{9}$ examined how task types and social contexts affect physiological arousal in MMORPG play. Results showed that when compared with solo play, collaborative play led to a significant decrease in arousal in response to violent tasks while leading to a slight increase for nonviolent tasks. Steinkuehler ${ }^{10}$ made use of ethnography to investigate the cultural practices and consequences of playing the MMORPG Lineage. Findings showed that through participation in activities within the MMORPG, gamers gained valuable knowledge about the customs and practices within Lineage. Gamers learned gaming terminology and gained knowledge of the terrain, the trading system, and the points system.

Chen et al. ${ }^{11}$ collected data on in-game player activities and observed how players join and leave guilds. Data were gathered for 641,805 avatars on 62 Taiwanese World of Warcraft servers. Chen et al. created five guild type categories that had different meanings in terms of in-game group dynamics. The guild types were newbie, small, large, elite, and unstable. These guild categories explained the guild life cycle (i.e., the formation of guilds, their membership and development), in-game mechanisms, and player behavior. Eatough et al. ${ }^{12}$ examined the perceptions of EverQuest players using interpretative phenomenological analysis (IPA). Data were analyzed from various online forums and showed that the gamers appeared to be "addicted" to EverQuest. Most of the gamers appeared to display the core components of addiction, such as salience, mood modification, tolerance, conflict, withdrawal symptoms, cravings, and relapse.

As has been observed in this brief overview, research into online gaming has used a variety of methods obtaining quantitative and qualitative data. Many of the quantitative 
studies can be criticized for obtaining small samples sizes, being culturally specific, and/or using pseudo-experimental methods in artificial settings recruiting students. There are many quantitative studies but very few qualitative studies. Further research focusing on the experiences of gamers that allows the gamers to speak and provide their own accounts of playing MMORPGs is needed. The present study explored the attitudes, experiences, and feelings of online gamers through qualitative interviews that made use of gamers' preferred methods of communicating (e-mail and instant messaging).

\section{Method}

\section{Participants}

Seventy-one online gamers (52 males, 19 females) participated in the study. The participants ranged in age from 18 to 54 years $(M=26$ years, $S D=8.4$ years $)$. Most of the participants were from the United States $(n=32)$, followed by the United Kingdom $(n=19)$, Canada $(n=5)$, and Netherlands $(n=4)$, although seven other countries (Norway, Sweden, Portugal, Finland, Australia, Belgium, and Greece) were also represented among the remaining participants $(n=11)$. The mean gaming time per week was 18.9 hours. The mean years of gaming experience was 4.7 years. Gamers were categorized into three gamer types: (a) casual gamer (plays 15 hours a week or less; $n=39$ ); (b) regular gamer (plays more than 15 hours and up to 30 hours a week; $n=21$ ); (b) excessive gamer (plays more than 30 hours a week; $n=12$ ).

\section{Design and procedure}

Participants were recruited in response to recruitment posts on various online gaming forums and in-game posts in the World of Warcraft MMORPG. The sample was therefore self-selecting. Participation was voluntary, and no incentive was offered for participation. Semi-structured interviews were conducted over a 4-month period (October 2007 to January 2008). The interviews were carried out (synchronously) via MSN Messenger (an online chat facility) or (asynchronously) via e-mail. All interviews were conducted at a time that was most convenient for the participants. The interviews began with a few structured questions regarding gamer demographics, then asked unstructured questions about gamers' feelings and experiences concerning their dayto-day playing behavior. The unstructured nature of the interviews allowed gamers to develop their own narrative by exploring their experiences of MMORPGs. The researcher allowed gamers to speak for themselves (i.e., the emergent themes were participant led rather than researcher led). This allowed gamers to take control of the interview process and prevented researchers' subjective bias entering the analytic stage. All participants were debriefed before and after the study. Each interview lasted approximately 75 minutes. Those who were interviewed by e-mail generally gave shorter responses to interviewer questions than did those interviewed using MSN Messenger. However, there was no difference in the type of response content. Data were gathered using the preferred medium of the gamer (i.e., e-mail or MSN Messenger). Data from the interviews were imported into QSR Nvivo, a qualitative data analysis software tool, and were analyzed using thematic analysis.
Thematic analysis is a flexible method for identifying, analyzing, and reporting themes within qualitative data. ${ }^{13}$ This method can offer a rich description of the data set and is very useful for summarizing large bodies of data. Thematic analysis also allows for the psychological interpretation of data and can generate unanticipated insights. In the first stage of the thematic analysis, the first author read through all the transcripts twice to become familiar with the data and then searched for the main themes to emerge from the responses to each of the questions asked. The responses were re-read with particular attention being paid to the themes arising from the first stage of data analysis. The responses were then collated under the emerging theme headings and were given provisional labels and definitions. The responses were again reread to see if they contained any further relevant information to the provisional themes. The themes were then given their final analytical form and definition and were refined further through systematic examination by the first and second authors. The searching, coding, and labeling of themes was done by the use of QSR Nvivo.

\section{Results}

Six main themes emerged from the analyses of the interview transcripts: (a) online gaming and integration into dayto-day lives; (b) online gaming, excessive play, and problems; (c) addiction; (d) psychosocial impact of online gaming; (e) online gaming, dissociation, and time loss; and (f) online gaming and the alleviation of negative feelings and mood states.

\section{Online gaming and integration into day-to-day lives}

The theme of online gaming and integration into day-today lives revealed how gamers fitted their online gaming into their lives. Many gamers said that they played in their free time after work, school, college, or university $(n=43 ; 33$ male, 10 female). Others said they integrated their online gaming by playing with friends $(n=3$, all male), while some said they played during work hours $(n=5 ; 4$ male, 1 female). A few gamers ( $n=3 ; 1$ male, 2 female) played with their spouses or romantic partners. Following are typical quotes by those who played in their free leisure time:

Extract 1: I leave for work at 7 a.m. and work 8 a.m.-5 p.m. I come home, help the kids with homework (I have teenagers), make sure the chores are done, have dinner, and then we log on after that. (P2, female, age 34, USA, regular gamer)

Extract 2: I play after my kids have gone to bed and I have "free" time to do as I wish. My wife chooses to watch TV; I choose to relax by playing video games. (P5, male, age 37, USA, excessive gamer)

These quotes show how gamers played at specific periods in their day-to-day lives. They played before or after work, after dinner, or after completing certain tasks. The findings indicated that gamers appeared to be very organized and managed their time very well. This is contrary to many beliefs that gamers lose control of their lives. Following are typical quotes of gamers who played with their friends:

Extract 3: WoW [World of Warcraft] takes a lot less priority now I'm in my final year at University. A couple of our friends who live nearby also play, so we try to arrange to meet up in 
game and complete an instance now and again. (P6, male, age 26, UK, casual gamer)

Extract 4: I play with friends I have in real life-both from home and meeting up with them online, and also going to their house and playing in the same room. (P7, male, age 27, USA, regular gamer)

These responses show how gamers played together with friends and how they made game playing a social activity they could enjoy together in real life or online. It also highlights the social nature of MMORPGs. Here are typical quotes of gamers who played while at work:

Extract 5: I work from home, so that doesn't pose a problem. My husband used to play with me, but quit. He went back to FFXI (Final Fantasy XI) and I play WoW (World of Warcraft). (P8, female, age 23, Canada, regular gamer)

Extract 6: I work from home, so if I don't have enough work to do I often spend the rest of the time playing MMORPGs. I also play after my work day is complete. (P9, male, age 36, USA, excessive gamer)

These quotes show how gamers did not have a problem with their jobs and playing MMORPGs. There was no real conflict between work and leisure time because they worked and played in the same location. Integrating online gaming into their lives was very simple for them. Following are some typical quotes from gamers who said they played with their spouses or romantic partners:

Extract 7: When at all possible, I play with my husband. We enjoy yelling insults at each other while our characters are interacting. Hardest part is finding the time to play while the kids are home. (P10, female, age 27, Canada, casual gamer)

Extract 8: I play with my partner, but this is because I actually met my partner through the game. We've since moved in together, but met online, and originally lived thousands of miles apart. (P11, female, age 22, USA, regular gamer)

These quotes show that gamers may play with their partners. For instance, P11 (Extract 8) originally met her partner online within the MMORPG. This shows how the MMORPG can be used as a medium to communicate and meet people.

\section{Online gaming, excessive play, and problems}

A majority of the gamers interviewed stated that their game playing is excessive ( $n=27 ; 20$ male, 7 female) or was excessive ( $n=13 ; 11$ male, 2 female). Only a few gamers said that their game playing was not excessive $(n=4 ; 2$ male, 2 female). The remainder of gamers did not comment on this issue. There was a high degree of awareness of game playing being excessive for those who said that they played excessively. For example, those whose current playing was excessive typically said the following:

Extract 9: Yes, [my playing is] very excessive. The end-game raiding content is what's keeping me hooked as well as the social aspect. (P15, male, age 18, UK, regular gamer)

Extract 10: I still feel I spend too much time playing-but sometimes I can't help myself. ... I just love building my gear and chatting. (P7, male, age 27, USA, regular gamer)

Extract 11: I really don't know why I play it so much. But it's where my friends are at, and it's just something to waste time with. I don't see myself as addicted to WoW [World of Warcraft], even though it seems that way. (P16, male, age 18, UK, excessive gamer)

These quotes show how the social aspects of MMORPGs can have the negative impact of causing excessive game playing. The virtual world was seen as a place to waste time, which led to excessive playing for some gamers. The online community also facilitated excessive play. Following are typical quotes from gamers who said their online gaming was (but no longer is) excessive:

Extract 12: It was quite excessive at one point. I wanted to try all the instances, try all the gear, level up as fast as possible. (P6, male, age 26, UK, casual gamer)

Extract 13: I was "raiding" in one of the best guilds in World of Warcraft. I felt I had the time for it, so I continued even if it required me to play around five to eight hours each day. So I have had a time it was excessive. (P17, male, age 21, Sweden, excessive gamer)

Extract 14: There were a couple of things that contributed to my excessive playing in the past. Foremost among them was belonging to a raiding guild. A second contributing factor was setting goals for myself. (P18, male, age 40, USA, regular gamer)

These quotes provide insight into the nature of MMORPGs and what caused many of the gamers to play excessively in the past. Gamers talked about the raiding, leveling up, and belonging to a guild as factors that caused excessive playing. Also, the quotes provide an insight into the thinking processes of the gamers (e.g., Extract 14, which explain parts of his gaming behavior and how he would tell himself that a specific quest had to be completed). There was a sense of urgency to complete the quest. Following are some quotes from gamers who said that their online gaming was not excessive:

Extract 15: I don't think my MMO playing is excessive. It's a hobby. (P19, female, age 20, USA, regular gamer)

Extract 16: Not really [game playing is not excessive], although I do play a lot. Most from a desire to finish what I started. (P21, male, age 21, UK, regular gamer)

Gamers here explained their online gaming behavior as not excessive and viewed it as a hobby. However, P21 (Extract 16) showed signs of playing excessively even though he said his game playing was not excessive.

\section{Addiction}

Online gaming was seen as potentially addictive by some gamers $(n=14 ; 10$ male, 4 female). For instance:

Extract 17: It's obvious that MMORPGs are addictive. My competitive nature is constantly hooked by testing my skill with other people. (P22, female, age 20, USA, casual gamer)

Extract 18: MMORPGs will be the new cocaine and the new trigger of the status of the mind's emotional state. MMORPGs are highly addictive. (P24, female, age 18, UK, regular gamer)

Extract 19: EverQuest used to be called "Evercrack" due to its highly addictive nature and large-scale social interactions. This led people to skip work to play, leading to a loss of a job (I watched it happen at my place of employment). (P25, male, age 27 , USA, regular gamer) 
These quotes highlight the potential addictiveness of MMORPGs as viewed by the players themselves. Social interaction, competition, and in-game tasks were some of the triggers to addiction, according to these gamers.

\section{Psychosocial impact of online gaming}

The vast majority of gamers ( $n=50 ; 35$ male, 15 female) highlighted the positive effects of online gaming, and many different aspects were touched on. However, a majority of the gamers ( $n=45$; 37 male, 8 female) also commented on the negative effects of MMORPGs. Positive aspects included:

Extract 20: You meet new people, you learn about new cultures, learn about teamwork, and build friendships. At the same time, you're having fun by playing a game. (P16, male, age $18, \mathrm{UK}$, excessive gamer)

Extract 21: I'm absolutely more proficient at [using] computers, I've made friendships that have stood the test of time. My husband and I are such a close unit now, and part of it is sharing a love for something that's so not the norm. (P2, female, age 34, USA, regular gamer)

Extract 22: MMORPGs can teach cooperation, typing, reading comprehension, economics, even complex mathematics. (P26, male, age 23, USA, casual gamer)

These quotes express some of the capabilities of MMORPGs. The gamers had many positive experiences with MMORPGs, and many other beneficial uses of MMORPGs were identified by participants. However, many of the gamers also noted potential negative effects:

Extract 23: I've lost my IRL [in real life] friends because I couldn't find the time to be with them, I quit school. Whenever someone asks me to do something on the weekends, I always think "Ooh, but we're raiding, I really shouldn't go out," and that's a way of thinking which I really dislike. (P32, female, age 21, Norway, excessive gamer)

Extract 24: Probably the most profound experience was losing friends to MMORPGs. I had a girlfriend who ended up playing 14 hours a day until I realised my attempts to help were futile and said goodbye. (P33, male, age 25, USA, regular gamer)

Extract 25: I have neglected hygiene, eating, sleep, work, and school because of it. It causes restlessness anytime I'm not playing. (P34, male, age 22, USA, casual gamer)

These quotes highlighted the potentially adverse effects that were associated with MMORPGs. Most notably, the experience of losing friends to MMORPGs is an issue that would appear to require further research.

\section{Online gaming, dissociation, and time loss}

Gamers were asked whether they experienced detachment from real life, whether they lost track of time, and whether they played longer than intended. Just under a third of gamers experienced detachment $(n=22 ; 17$ male, 5 female). Over a third of gamers experienced time loss and said they played longer than intended ( $n=25 ; 16$ male, 9 female). In terms of detachment, gamers reported:

Extract 26: I noticed what a mess my bedroom is. I do the dishes every morning, that's about the only chore I have. I put myself last. If I need to do something in-game, I'll wait with the shower and the dinner. (P32, female, age 21, Norway, excessive gamer)

Extract 27: When I'm playing online, I don't even think of me as sitting in the same world as everyone else. I get sucked into the game and the only thing that matters to me is that game. (P22, female, age 20, USA, casual gamer)

Extract 28: I do actually [feel detachment]. I feel that [other tasks] can wait and that I can do them later, which results in time being wasted and usually me not doing the homework or my household tasks given to me. (P29, male, age 21, USA, regular gamer)

These quotes showed the immersive properties of MMORPGs that caused detachment in some gamers. This appeared to be an appealing feature of MMORPGs. Of those who played longer than intended, typical quotes included:

Extract 29: One doesn't always realise how long one has played (as witness the occasional Friday night when I suddenly realise it's 3 a.m., and should have gone to bed hours ago). Game time is different (time is not 1:1), it is easy to fail to notice physical time. (P27, female, age 40, USA, casual gamer)

Extract 30: I do tend to lose track of time while playing. WoW is an incredibly immersive game and I become very absorbed in what my character is trying to accomplish. (P37, female, age 33, Canada, casual gamer)

Extract 31: I do lose time when I am online gaming. I would think that an hour went by and it was like 4 [or] 5. I started setting an alarm clock for every hour so it would help me keep track of time. (P12, male, age 33, USA, casual gamer)

The quotes above show that gamers experienced time loss to such an extent that some gamers set an alarm to alert them of the time. The level of involvement that is required when playing a MMORPG was seen as a reason for time loss.

\section{Online gaming and the alleviation of negative feelings and mood states}

Analysis demonstrated how gamers used online gaming to alleviate negative feelings that in turn brought positive feelings. Just under a third of gamers $(n=22 ; 13$ male, 9 female) spoke about how they removed negative feelings such as stress, anger, and frustration by playing MMORPGs. For instance:

Extract 32: I usually use WoW to relieve loneliness by finding my friends or making new ones. It fills you with happiness that you are having a great time even when no one is around you. (P24, female, age 18, UK, regular gamer)

Extract 33: I have used [MMORPGs] to alleviate frustration. It is much better to go and kill monsters than to yell at the family. They can be a good release. (P38, female, age 54, USA, regular gamer)

Extract 34: For me it's a way of stepping out of everyday stress and annoyances and entering a sort of sanitised world where I am mostly in control of what happens to me. (P41, female, age 27 , UK, casual gamer)

These quotes showed how gamers utilized MMORPGs to relieve very strong negative feelings that can be difficult to relieve in some cases. Gamers used MMORPGs as a release and as a medium to "step away" away from everyday problems. 


\section{Discussion}

The study examined whether online gaming impacts (psychologically and socially) on peoples' lives by exploring attitudes and views toward online game playing and consequent behavior. The qualitative nature of the study enabled an in-depth analysis of gamers' perceptions. The interviews allowed for the development of qualitative accounts that provided information about particular aspects of online gaming. For instance, the study showed how gamers used MMORPGs to alleviate negative feelings, and it provided detailed descriptions of personal problems that arose due to playing MMORPGs. The study also provided detailed accounts of gamers' day-to-day lives and how they integrated online gaming into their lives.

\section{Online gaming and integration into day-to-day lives}

The results showed that more than half of gamers $(n=43)$ played MMORPGs in their free time outside of school, college, or university, while a smaller number $(n=5)$ played while at work. These findings show that gamers integrate online gaming into their lives with few adverse social effects on their daily lives. Results also showed that most gamers planned their gaming around their daily education, work, and/or home tasks. For instance, one gamer reported that he played after his children had gone to bed (Extract 2). The results show that gamers tend to manage and integrate their MMORPG playing into their lives but that a few play along with friends and partners. These findings were similar to those of Cole and Griffiths, ${ }^{5}$ who found that $26 \%$ of their sample played MMORPGs with family and real-life friends. The findings also show similarity to that of Whang and Chang, ${ }^{14}$ who explored the lifestyles of online gamers and compared their real-world lifestyles with their values and attitudes in the virtual world.

\section{Online gaming, excessive play, and problems}

The present study also found that more than half of the gamers $(n=40)$ thought their game playing is or was excessive. Only four gamers specifically said that their game playing was not excessive. The reasons for excessive play were varied. For instance, one gamer (Extract 9) said the "end-game raiding" content as well as the social aspects of the game caused him to play excessively. Another gamer (Extract 10) stated that the social aspect as well as "building his gear" caused gaming excess. Other reasons for excessive playing included the competitive surroundings of the virtual environment, the community, having a lot of free time, the lack of interesting alternative entertainment outlets with the same relative ease of coming and going, and having to get their money's worth if they had paid a subscription. For those who said their game playing was excessive in the past, they provided similar responses to those who played excessively at present.

The gamers who said that their playing was not excessive saw their online gaming as a hobby (e.g., Extract 15). From the findings, online gaming appeared to facilitate excessive play to a high extent. The findings showed that gamers were aware of their game-playing behavior, and for many, their playing was excessive. Specific aspects of MMORPGs were highlighted as causing excessive play that could lead to ad- verse health effects for some gamers (such as missing meals and not exercising regularly). Future research should try to assess the extent of excessive playing using a quantitative measure. There were also some insights into online gaming as an addiction. A fifth of gamers $(n=14)$ thought MMORPGs were addictive. One gamer (Extract 18) likened MMORPGs to a drug and thought they were highly addictive. Another gamer (Extract 17) saw her playing as excessive and got "hooked" by testing her skill with other people. These findings support those of Grusser et al., ${ }^{7}$ who found that $12 \%$ of gamers met at least three addiction criteria. However, the present study does not claim that the gaming behavior of the participants is related to dysfunctional social behavior. Conversely, in regard to the present findings, the qualitative data on excessive playing and addiction needs to be supported with quantitative data.

\section{Psychosocial impact of online gaming}

The present study was distinctive in terms of collecting data on the positive and negative effects of online gaming. More than two thirds of gamers $(n=51)$ commented on the positive effects of online gaming. Some of the positive effects included meeting new people, learning about new cultures, facilitating teamwork, and building friendships (Extract 20). Online gaming was linked to improved efficiency at using computers (Extract 21). It was also seen as a good tool for teaching cooperation, typing, reading comprehension, economics, and mathematics (Extract 22). Interestingly, online gaming was also seen as a medium that allowed people to exercise their imagination and could teach teamwork and planning skills. Such positive effects lend support to the collaborative learning approaches that focus on problem-based and experienced-based learning and to the literature showing online games can be educationally useful. ${ }^{16}$

The negative effects of online gaming were commented on by almost two thirds of gamers $(n=45)$. Somewhat paradoxically, gamers linked online gaming to being social online but antisocial in real life. Other negative effects included online gaming being time consuming and affecting the breakdown of friendships and relationships (Extract 23). One gamer (Extract 25) provided detailed information of how he had neglected hygiene, eating, sleep, work, and school due to playing online games. Video game developers could benefit from these findings by incorporating more of the positive aspects and omitting the negative aspects when developing new MMORPGs. For instance, MMORPGs could incorporate rest breaks and reward gamers for doing so. In-game quests could perhaps be made to be more educationally oriented. Rather than making tasks that are repetitive, tasks that test gamers' analytical, communication, and decision-making skills would be one way of making the games more educational.

\section{Online gaming, dissociation, and time loss}

The present study also examined the potential capabilities of online gaming to cause detachment and/or time loss. Less than a third of gamers $(n=22)$ said that they experienced detachment. For one gamer (Extract 29) the experience of detachment had caused her to delay real-world tasks such as cleaning her bedroom and taking showers. Another gamer 
(Extract 30) thought she was in a different world from everyone else. She also mentioned the feeling of getting "sucked" into the game. These accounts detail the types of feelings and thoughts that were provoked by online gaming. Just over a third of gamers $(n=25)$ stated that they experienced time loss and played longer than intended. Participants' comments were varied on this topic. One gamer (Extract 31) felt that time went by quickly, and she mentioned that in-game time is different from real-world time and can lead to not noticing real-world time. Others experienced time loss and resorted to setting an alarm. These findings support the findings of Wood et al. ${ }^{17}$ that showed that gamers experienced time loss, which was associated with particular structural characteristics of video games, such as their multiplayer interaction, multiple levels, and their complexity. Further research on both time loss and its link with particular structural characteristics, such as that by Wood et al., ${ }^{18}$ is needed.

\section{Online gaming and the alleviation of negative feelings and mood states}

The interviews also yielded data on how gamers used online gaming to alleviate negative feelings. Almost one third of gamers $(n=22)$ spoke about such effects. Gamers used online gaming to relieve loneliness and boredom and also to alleviate frustration (Extract 33). Online games were used to relieve everyday stresses and annoyances. Here, online gaming had a therapeutic capability and could perhaps be used in psychotherapy by allowing patients to play MMORPGs in order to relieve negative feelings. Hence, online games appear to have the capability to bring about positive behavioral changes, as noted in past video game research in psychotherapeutic settings. ${ }^{19}$ However, further research that examines the psychological and therapeutic aspects of online games is needed.

This study did not set out to find findings that can be generalized. It was a qualitative study and thus emphasized the gathering of rich, elaborate, meaningful data. The semistructured interviews conducted via the use of MSN Messenger and e-mail provided a more in-depth analysis of gamers perceptions of online gaming. This method can be seen to have many advantages. For instance, instant messaging interviews are cheap to administer, obtain geographically diverse samples, and are less time consuming compared to face-to-face interviews. Research by Stieger and Goritz ${ }^{20}$ showed that instant message interviews were a feasible method of data collection, as the quality of data, the contact rate, response rate, and retention rate was good. They also found that the risk of obtaining false responses in instant message interviews was small.

There are some limitations associated with the use of instant messaging and e-mail interviewing. The mixture of synchronous and asynchronous data collection may have influenced the findings. Furthermore, nonverbal (e.g., body language) and paralinguistic (e.g., tone of voice) cues were not available, making it difficult for the interviewer to know the psychological disposition of the interviewees. However, technologies exist that allow the incorporation of audio and video capabilities into online real-time chat (e.g., webcams), thus making the setting resemble a face-to-face interview setting. ${ }^{40}$ However, there are technical difficulties associated with incorporating audio and video into online interviews, such as interruptions due to loss of connections or slow data transmission rates. The interview questions were loose and unstructured, allowing the gamers to talk about what was important to them rather than what we, as researchers, felt were the salient issues. Therefore, some of the themes that emerged were discussed by only a minority of gamers.

The online interview method used in this study appeared to lead to enhanced levels of candidness from gamers, as can be seen in the quote from one gamer (Extract 23) who provided detailed information about her social life. The anonymity provided by this method could also explain the willingness of gamers to disclose highly personal and sensitive information. The lack of nonverbal and paralinguistic cues may have contributed to the high levels of self-disclosure. Furthermore, past online research has shown high levels of self-disclosure from participants and reduced levels of social desirable responses. ${ }^{21}$ The present study revealed a variety of attitudes and experiences of gamers. It showed the positive and negative effects of online games, gender differences, and online and offline use of gaming technologies by gamers. It can be seen that the instant message interview and e-mail interview are feasible methods for data collection. The qualitative accounts of gamers prove the suitability of such methods for further research.

\section{Disclosure Statement}

No competing financial interests exist.

\section{References}

1. Griffiths MD, Davies MNO, Chappell D. Breaking the stereotype: the case of online gaming. CyberPsychology \& Behavior 2003; 6:81-91.

2. Griffiths MD, Davies MNO, Chappell D. Demographic factors and playing variables in online computer gaming. CyberPsychology \& Behavior 2004; 7:487-95.

3. Yee N. The demographics, motivations and derived experiences of users of massively multi-user online graphical environments. Presence 2006; 15:309-29.

4. Chen C, Sun C, Hsieh J. Player guild dynamics and evolution in massively multiplayer online games. CyberPsychology \& Behavior 2008; 11:293-301.

5. Cole H, Griffiths MD. Social interactions in massively multiplayer online role-playing games. CyberPsychology \& Behavior 2007; 10:575-83.

6. Yee N. Motivations for play in online games. CyberPsychology \& Behavior 2006; 9:772-5.

7. Grusser SM, Thalemann R, Griffiths MD. Excessive computer game playing: evidence for addiction and aggression? CyberPsychology \& Behavior 2007; 10:290-2.

8. Hussain Z, Griffiths MD. Gender swapping and socializing in cyberspace: an exploratory study. CyberPsychology \& Behavior 2008; 11:47-53.

9. Lim S, Lee JR. When playing together feels different: effects of task types and social contexts on physiological arousal in multiplayer online gaming contexts. CyberPsychology \& Behavior 2009; 12:59-61.

10. Steinkuehler C. Massively multiplayer online gaming as a constellation of literacy practices. eLearning 2007; 4:297-318. 
11. Chen C, Sun C, Hsieh J. Player guild dynamics and evolution in massively multiplayer online games. CyberPsychology \& Behavior 2008; 11:293-301.

12. Eatough V, Davies MNO, Griffiths MD, et al. EverQuestit's just a computer game right? An interpretative phenomenological analysis of online gaming addiction. International Journal of Mental Health \& Addiction 2006; 4:205-16.

13. Braun V, Clarke V. Using thematic analysis in psychology. Qualitative Research in Psychology 2006; 3:77-101.

14. Whang LS, Chang G. Lifestyles of virtual world residents: living in the online game "lineage." CyberPsychology \& Behavior 2004; 7:592-600.

15. Wan C, Chiou W. The motivations of adolescents who are addicted to online games: a cognitive perspective. Adolescence 2007; 42:181-97.

16. de Freitas S, Griffiths MD. (2008) Massively multiplayer online role-play games for learning. In Ferdig RE, ed. Handbook of research on effective electronic gaming in education. pp. 51-65. Hershey: Information Science Reference.

17. Wood RTA, Griffiths MD, Parke A. Experiences of time loss among videogame players: an empirical study. CyberPsychology \& Behavior 2007; 8:38-44.
18. Wood RTA, Griffiths MD, Chappell D, et al. The structural characteristics of video games: a psycho-structural analysis. CyberPsychology \& Behavior 2004; 7:1-10.

19. Gardner JE. Can the Mario Bros. help? Nintendo games as an adjunct in psychotherapy with children. Psychotherapy 1991; 28:667-70.

20. Stieger S, Goritz A. Using instant messaging for Internetbased interviews. CyberPsychology \& Behavior 2006; 9:552-9.

21. Joinson A. Social desirability, anonymity and Internet-based questionnaires. Behavior Research Methods, Instruments \& Computers 1999; 31:433-8.

Address correspondence to: Zaheer Hussain International Gaming Research Unit Psychology Division, Department of Social Sciences Nottingham Trent University Burton Street Nottingham, NG1 4BU United Kingdom

E-mail: zaheer.hussain@ntu.ac.uk 
